\title{
The first two transient supersoft X-ray sources in M 31 globular clusters and the connection to classical novae ${ }^{\star}$
}

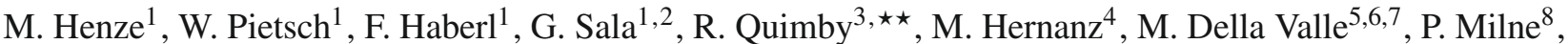 \\ G. G. Williams ${ }^{8}$, V. Burwitz ${ }^{1}$, J. Greiner ${ }^{1}$, H. Stiele ${ }^{1}$, D. H. Hartmann ${ }^{9}$, A. K. H. Kong ${ }^{10}$, and K. Hornoch ${ }^{11}$ \\ 1 Max-Planck-Institut für extraterrestrische Physik, 85748 Garching, Germany \\ e-mail: mhenze@mpe.mpg.de \\ 2 Departament de Física i Enginyeria Nuclear, EUETIB (UPC/IEEC), Comte d'Urgell 187, 08036 Barcelona, Spain \\ 3 University of Texas, Austin TX, 78712, USA \\ 4 Institut de Ciències de l'Espai (CSIC-IEEC), Campus UAB, Fac. Ciències, 08193 Bellaterra, Spain \\ 5 European Southern Observatory (ESO), 85748 Garching, Germany \\ 6 INAF-Napoli, Osservatorio Astronomico di Capodimonte, Salita Moiariello 16, 80131 Napoli, Italy \\ 7 International Centre for Relativistic Astrophysics, Piazzale della Repubblica 2, 65122 Pescara, Italy \\ 8 Steward Observatory, 933 North Cherry Avenue, Tucson, AZ 85721, USA \\ 9 Department of Physics and Astronomy, Clemson University, Clemson, SC 29634-0978, USA \\ 10 Institute of Astronomy and Department of Physics, National Tsing Hua University, Hsinchu, Taiwan \\ 11 Astronomical Institute, Academy of Sciences, 25165 Ondřejov, Czech Republic
}

Received 9 October 2008 / Accepted 21 April 2009

\section{ABSTRACT}

Context. Classical novae (CNe) have been found to represent the major class of supersoft X-ray sources (SSSs) in our neighbour galaxy M 31.

Aims. We determine the properties and evolution of the two first SSSs ever discovered in the M 31 globular cluster (GC) system. Methods. We have used XMM-Newton, Chandra and Swift observations of the centre region of M 31 to discover both SSSs and to determine their X-ray light curves and spectra. We performed detailed analysis of XMM-Newton EPIC PN spectra of the source in Bol 111 (SS1) using blackbody and NLTE white dwarf (WD) atmosphere models. For the SSS in Bol 194 (SS2) we used optical monitoring data to search for an optical counterpart.

Results. Both GC X-ray sources were classified as SSS. We identify SS1 with the CN M31N 2007-06b recently discovered in the M 31 GC Bol 111. For SS2 we did not find evidence for a recent nova outburst and can only provide useful constraints on the time of the outburst of a hypothetical nova.

Conclusions. The only known CN in a M 31 GC can be identified with the first SSS found in a M 31 GC. We discuss the impact of our observations on the nova rate for the M $31 \mathrm{GC}$ system.

Key words. galaxies: individual: M 31 - novae, cataclysmic variables - stars: individual: Nova M31N 2007-06b globular clusters: individual: Bol 111 - globular clusters: individual Bol 194 - X-rays: galaxies

\section{Introduction}

Supersoft X-ray sources (SSSs) are a class of X-ray sources that were first characterised based on ROSAT observations (see e.g. Greiner et al. 1991). These sources show extremely soft X-ray spectra, with little or no radiation at energies above $1 \mathrm{keV}$ (see e.g. Parmar et al. 1998), that can be described by blackbody temperatures typically in the range of $15-80 \mathrm{eV}$ (see Kahabka \& van den Heuvel 1997, and references therein). SSSs were originally believed to be hydrogen burning white dwarfs (WDs) in binary systems, where the WD steadily burns hydrogen rich matter accreted from its companion star (Kahabka \& van den Heuvel 1997). However, the class of SSSs is quite inhomogeneous. Prototypical sources are, on the one hand, CAL 83 (see Parmar et al. 1998, and references therein) and CAL 87 (see Parmar et al. 1997, and references therein), both of which are located in the

\footnotetext{
* Partly based on observations with XMM-Newton, an ESA Science Mission with instruments and contributions directly funded by ESA Member States and NASA.

$\star \star$ Current address: California Institute of Technology, Pasadena, CA 91125, USA.
}

Large Magellanic Cloud. These objects show eclipses (CAL 87) or rare X-ray off states (CAL 83) (Kahabka \& van den Heuvel 1997), but are rather permanent SSSs. On the other hand, Pietsch et al. (2005a) found out that classical novae $(\mathrm{CNe})$ represent the major class of SSSs in our neighbour galaxy M 31 (distance 780 kpc, Holland 1998; Stanek \& Garnavich 1998). CNe can appear as luminous $\left(L \sim 10^{38} \mathrm{erg} \mathrm{s}^{-1}\right)$ transient SSSs that seem to go through a single outburst that can last from months to several years (Pietsch et al. 2007b).

Classical novae $(\mathrm{CNe})$ are thermonuclear explosions on the surface of WDs in cataclysmic binaries that result from the transfer of matter from the companion star to the WD. The transferred hydrogen-rich matter accumulates on the surface of the WD until hydrogen ignition starts a thermonuclear runaway in the degenerate matter of the WD envelope. The resulting expansion of the hot envelope can cause the brightness of the WD to rise by more than nine magnitudes within a few days, and mass to be ejected at high velocities (see Hernanz 2005; Warner 1995, and references therein). However, a fraction of the hot envelope can remain in steady hydrogen burning on the surface of the WD (Starrfield et al. 1974; Sala \& Hernanz 2005), 
powering a supersoft X-ray source that can be observed directly once the ejected envelope becomes sufficiently transparent (Starrfield 1989; Krautter 2002).

The duration of the supersoft phase is related to the amount of H-rich matter that is not ejected and also depends on the luminosity of the white dwarf. More massive WDs need to accrete less matter to initiate the thermonuclear runaway, because of their higher surface gravity (Jose \& Hernanz 1998). In general, more massive WDs retain less accreted mass after the explosion, although this also depends on the accretion rate, and reach larger luminosities (Yaron et al. 2005). Thus, the duration of the SSS state is inversely related to the mass of the WD (Sala \& Hernanz 2005; Tuchman \& Truran 1998). In turn, the transparency requirement mentioned above implies that the time of appearance of the SSS is determined by the fraction of mass ejected in the outburst (Hachisu \& Kato 2006). X-ray lightcurves therefore provide important clues on the physics of the nova outburst, addressing the key question whether a WD accumulates matter over time to become a potential progenitor for a type Ia supernova (SN-Ia).

Due to its proximity to the Galaxy and its moderate Galactic foreground absorption $\left(N_{\mathrm{H}}=0.7 \times 10^{21} \mathrm{~cm}^{-2}\right.$, Stark et al. 1992), M 31 is a unique target for CN surveys. Starting with Hubble (1929) the majority of these surveys that were conducted in the past (see e.g. Henze et al. 2008b, and references therein) classified optical transients as novae just by their lightcurve. Following the steep rise, the luminosity of different $\mathrm{CNe}$ declines with different speed, which allows a phenomenological classification of these objects by their speed class (Payne-Gaposchkin 1964). Eventually, novae in M 31 will fade back to invisibility, since the binary systems in quiescence are too faint to be observed at the distance of M 31. Only recently, nova monitoring programs for M 31 were established, that include fast data analysis and that therefore provide the possibility to conduct follow-up spectroscopy (see e.g. Henze et al. 2008a; Di Mille et al. 2008) and to classify CNe within the system of Williams (1992).

Almost all optical surveys for CNe in M 31 that were conducted in the past searched for suddenly appearing objects that have not been visible before and fade back to invisibility in days to weeks. This condition is certainly not fulfilled by CNe in relatively bright GCs, where the optical background light of the GC itself makes a photometric discovery of a nova outburst much more complicated. Therefore, the connection of CNe to SSSs in $\mathrm{X}$-rays provides a useful possibility to detect $\mathrm{CNe}$ in GCs. Novae in GCs are rare. There were just two sources known so far that likely fit this definition. One of them was seen in the Galactic GC M 80 whereas the second nova was found in a GC of the galaxy M 87 (see Shara et al. 2004, and references therein). According to Shara et al. (2004) a third candidate (nova 1938 in the galactic GC M 14) is less likely to be a GC nova. Recently, Shafter \& Quimby (2007) reported the very first nova found in a M 31 GC (M31N 2007-06b).

Independent from their connection to $\mathrm{CNe}, \mathrm{SSSs}$ in GCs are rare objects. There was just one SSS known in GC up to now: the transient 1E 1339.8+2837 in the Galactic GC M 3 (NGC 5272) (Dotani et al. 1999; Verbunt et al. 1995). 1E 1339.8+2837 was first discovered with the Einstein satellite with a hard X-ray spectrum and a low luminosity of $4 \times 10^{33} \mathrm{erg} \mathrm{s}^{-1}$ (Hertz \& Grindlay 1983) but showed a very soft high luminosity state during 1991-92 when observed with ROSAT (Dotani et al. 1999). Dotani et al. (1999) give for 1E 1339.8+2837 a blackbody temperature of $k T \sim 36 \mathrm{eV}$ and a luminosity of $\sim 10^{35} \mathrm{erg} \mathrm{s}^{-1}$, which is significantly lower than the observed peak luminosities of other SSS, including the two sources discussed in this work. They discuss this object as a cataclysmic variable (CV) system that may be a dwarf nova including a massive WD. The CV interpretation is supported by Edmonds et al. (2004) who used Hubble Space Telescope observations to discover an optical counterpart to $1 \mathrm{E} 1339.8+2837$. They suggest that magnetically channeled accretion could explain the peculiarities of $1 \mathrm{E} 1339.8+2837$.

Recently, we reported, based on preliminary data analysis (Pietsch et al. 2007a), the very first SSS (hereafter SS1) that was found in a M 31 GC. During the same observing run where we found this source we detected a second luminous and previously unknown SSS (hereafter SS2) in another M 31 GC (Haberl et al. 2007a,b). Both sources are transients and will be examined in detail in this work.

The structure of the paper is as follows: in Sect. 2 we describe our X-ray observations and the properties of the two SSSs. The optical data available for Bol 194 and the data analysis are presented and interpreted in Sect. 3. Finally, in Sect. 4 we summarise our results and discuss the impact that two GC novae within one year would have on the nova rate for the M $31 \mathrm{GC}$ system.

\section{X-ray observations and data analysis}

In the context of the XMM-Newton/Chandra M 31 nova monitoring project ${ }^{1}$ we obtained five $20 \mathrm{ks}$ Chandra HRC-I observations of M31 starting in November 2007. We detected two new sources in the M31 GCs Bol 111 and Bol 194 (Galleti et al. 2004). For both sources follow-up ToO observations with the Swift X-ray Telescope (XRT) were requested (e.g. Pietsch et al. 2007a; Kong \& Di Stefano 2007b) as well as additional Chandra ACIS-S observations by another group (Galache et al. 2007). We also used two observations obtained within the XMM-Newton M 31 large survey project ${ }^{2}$. Details on the observations used are given in Tables 1 and 2 for the sources in Bol 111 and Bol 194, respectively. The tables list the telescopes and instruments used, the observation identifications (ObsIDs), the dates as well as source count rates and luminosities or upper limits, respectively. X-ray light curves of both sources are shown in Fig. 1. Figure 2 shows the fields of view of Chandra and XMM-Newton observations with representative positions.

We analysed all available observations using mission dependent source detection software as well as the HEAsoft package v6.3, including the spectral analysis software XSPEC v12.3.1. In all our XSPEC models we used the Tübingen-Boulder ISM absorption (TBabs in XSPEC) model together with the photoelectric absorption cross-sections from Balucinska-Church \& McCammon (1992) and ISM abundances from Wilms et al. (2000). For the individual telescopes we applied the following data reduction techniques:

The Swift XRT data were analysed using the HEAsoft XIMAGE package (version 4.4) with the sosta command (source statistics) for estimations of count rates. We took into account the XRT PSF of the sources that we computed with the command psf, as well as exposure maps that were created

\footnotetext{
1 http://www .mpe.mpg.de/ m31novae/xray/index.php

2 XMM-Newton Large Program: the X-ray source population of the Andromeda galaxy M 31; PI: W. Pietsch.

See

http://www .mpe.mpg.de/xray/research/normal_galaxies/ m31/lp.php
} 
Table 1. X-ray observations of SS1 in Bol 111.

\begin{tabular}{|c|c|c|c|c|c|c|}
\hline Telescope/Instrument ${ }^{a}$ & ObsID & $\begin{array}{r}\text { Exp. time }{ }^{b} \\
{[\mathrm{ks}]}\end{array}$ & $\begin{array}{l}\text { Date }^{c} \\
{[\mathrm{UT}]}\end{array}$ & $\begin{array}{r}\text { Offset }^{d} \\
{[\mathrm{~d}]}\end{array}$ & $\begin{array}{r}\text { Count Rate } \text { Rat }^{e} \\
{\left[\mathrm{ct} \mathrm{s}^{-1}\right]}\end{array}$ & $\begin{array}{r}\mathrm{L}_{0.2-1.0^{e}} \\
{\left[\mathrm{erg} \mathrm{s}^{-1}\right]}\end{array}$ \\
\hline XMM-Newton EPIC PN & 0505760201 & 49.2 & $2007-07-22.55$ & 33 & $<1.1 \times 10^{-3}$ & $<1.4 \times 10^{36}$ \\
\hline $\begin{array}{l}\text { Chandra } \text { HRC-I } \\
\text { Chandra } \text { HRC-I } \\
\text { Chandra } \text { HRC-I } \\
\text { Chandra HRC-I } \\
\text { Chandra } \text { HRC-I }\end{array}$ & $\begin{array}{l}8526 \\
8527 \\
8528 \\
8529 \\
8530 \\
\end{array}$ & $\begin{array}{l}18.7 \\
20.0 \\
20.0 \\
18.9 \\
19.9 \\
\end{array}$ & $\begin{array}{l}2007-11-07.64 \\
2007-11-17.76 \\
2007-11-28.79 \\
2007-12-07.57 \\
2007-12-17.49 \\
\end{array}$ & $\begin{array}{l}141 \\
151 \\
162 \\
171 \\
181 \\
\end{array}$ & $\begin{array}{r}>(1.9 \pm 0.4) \times 10^{-2} \\
(2.5 \pm 0.2) \times 10^{-2} \\
(2.2 \pm 0.2) \times 10^{-2} \\
(2.8 \pm 0.3) \times 10^{-2} \\
(2.5 \pm 0.3) \times 10^{-2}\end{array}$ & $\begin{array}{r}>(5.3 \pm 1.1) \times 10^{38} \\
(7.0 \pm 0.7) \times 10^{38} \\
(6.0 \pm 0.6) \times 10^{38} \\
(7.7 \pm 0.7) \times 10^{38} \\
(7.1 \pm 0.7) \times 10^{38}\end{array}$ \\
\hline $\begin{array}{l}\text { Swift XRT } \\
\text { Swift XRT } \\
\text { Swift XRT } \\
\text { Swift XRT } \\
\text { Swift XRT } \\
\text { Swift XRT } \\
\text { Swift XRT } \\
\text { Swift XRT } \\
\text { Swift XRT } \\
\text { Swift XRT } \\
\text { Swift XRT } \\
\end{array}$ & $\begin{array}{r}00031017001 / 2 \\
00031017003 \\
00031017004 \\
00031017005 \\
00031017006 \\
00031017007 \\
00031017008 \\
00031017009 \\
00031017010 \\
00031017011 \\
00031017012 \\
\end{array}$ & $\begin{array}{l}7.1 \\
3.0 \\
3.0 \\
3.2 \\
2.2 \\
2.1 \\
2.3 \\
2.3 \\
2.0 \\
1.9 \\
1.7 \\
\end{array}$ & $\begin{array}{l}2007-11-18.40 \\
2007-11-13.02 \\
2007-12-14.02 \\
2007-12-15.03 \\
2007-12-20.25 \\
2007-12-22.39 \\
2007-12-24.33 \\
2007-12-30.15 \\
2008-01-03.44 \\
2008-01-06.25 \\
2008-01-10.00 \\
\end{array}$ & $\begin{array}{l}152 \\
177 \\
178 \\
179 \\
184 \\
186 \\
188 \\
194 \\
198 \\
201 \\
205 \\
\end{array}$ & $\begin{array}{r}(1.18 \pm 0.15) \times 10^{-2} \\
(0.8 \pm 0.2) \times 10^{-2} \\
(1.1 \pm 0.2) \times 10^{-2} \\
(1.1 \pm 0.2) \times 10^{-2} \\
(1.1 \pm 0.3) \times 10^{-2} \\
(0.5 \pm 0.2) \times 10^{-2} \\
(0.9 \pm 0.2) \times 10^{-2} \\
(1.0 \pm 0.2) \times 10^{-2} \\
(1.2 \pm 0.3) \times 10^{-2} \\
(0.4 \pm 0.3) \times 10^{-2} \\
(1.1 \pm 0.3) \times 10^{-2} \\
\end{array}$ & $\begin{aligned}(11.6 \pm 1.4) & \times 10^{38} \\
(8.1 \pm 1.9) & \times 10^{38} \\
(10.6 \pm 2.2) & \times 10^{38} \\
(11.1 \pm 2.1) & \times 10^{38} \\
(11.1 \pm 2.6) & \times 10^{38} \\
(5.1 \pm 1.9) & \times 10^{38} \\
(9.1 \pm 2.4) & \times 10^{38} \\
(10.0 \pm 2.4) & \times 10^{38} \\
(12.2 \pm 2.8) & \times 10^{38} \\
(4.0 \pm 2.8) & \times 10^{38} \\
(11.1 \pm 1.3) & \times 10^{38}\end{aligned}$ \\
\hline $\begin{array}{l}\text { XMM-Newton EPIC PN } \\
\text { XMM-Newton EPIC PN } \\
\text { Swift XRT }\end{array}$ & $\begin{array}{r}0511380201 \\
0511380601 \\
00037718001 \\
\end{array}$ & $\begin{array}{r}23.0 \\
24.0 \\
4.8 \\
\end{array}$ & $\begin{array}{l}2008-01-05.99 \\
2008-02-09.31 \\
2008-05-26.29 \\
\end{array}$ & $\begin{array}{l}200 \\
235 \\
342 \\
\end{array}$ & $\begin{array}{l}(8.2 \pm 0.2) \times 10^{-2} \\
(7.5 \pm 0.2) \times 10^{-2} \\
(0.5 \pm 0.1) \times 10^{-2} \\
\end{array}$ & $\begin{array}{r}(10.6 \pm 0.2) \times 10^{38} \\
(12.2 \pm 0.4) \times 10^{38} \\
(4.6 \pm 1.3) \times 10^{38}\end{array}$ \\
\hline XMM-Newton EPIC PN & 0560180101 & 17.4 & $2008-07-18.26$ & 395 & $(3.0 \pm 0.2) \times 10^{-2}$ & $(8.9 \pm 0.5) \times 10^{38}$ \\
\hline
\end{tabular}

Notes: ${ }^{a}$ Telescope and instrument used for observation.

${ }^{b}$ Dead time corrected exposure time of the observation.

${ }^{c}$ Start date of the observation.

${ }^{d}$ Time in days after the discovery of nova M31N 2007-06b in the optical (Shafter \& Quimby 2007) on 2007 June 19.38. $(\mathrm{JD}=2454271)$.

${ }^{e}$ Source count rates, X-ray luminosities (unabsorbed, blackbody fit, $0.2-1.0 \mathrm{keV}$ ) and upper limits were estimated according to Sect. 2.1. For Chandra ObsID 8526 the source is right on the detector edge, therefore we give lower luminosity limits.

Table 2. X-ray observations of SS2 in Bol 194.

\begin{tabular}{|c|c|c|c|c|c|c|}
\hline Telescope/Instrument ${ }^{a}$ & ObsID & $\begin{array}{r}\text { Exp. time }^{b} \\
{[\mathrm{ks}]} \\
\end{array}$ & $\begin{array}{c}\text { Date }^{c} \\
\text { [UT] } \\
\end{array}$ & $\begin{array}{r}\text { Offset }^{d} \\
{[\mathrm{~d}]} \\
\end{array}$ & $\begin{array}{r}\text { Count Rate } e^{e} \\
{\left[\mathrm{ct} \mathrm{s}^{-1}\right]}\end{array}$ & $\begin{array}{r}\text { Luminosity }^{e} \\
{\left[\mathrm{erg} \mathrm{s}^{-1}\right]}\end{array}$ \\
\hline XMM-Newton EPIC PN & 0505760201 & 49.2 & $2007-07-22.55$ & 0 & $<2.9 \times 10^{-3}$ & $<1.6 \times 10^{36}$ \\
\hline Chandra ACIS-S & 8186 & 5.0 & 2007-11-03.18 & 104 & $2.1 \times 10^{-2}$ & $3.4 \times 10^{37}$ \\
\hline Chandra HRC-I & 8526 & 18.7 & 2007-11-07.64 & 108 & $(3.55 \pm 0.25) \times 10^{-2}$ & $(8.41 \pm 0.59) \times 10^{37}$ \\
\hline Chandra HRC-I & 8527 & 20.0 & 2007-11-18.76 & 118 & $(2.95 \pm 0.25) \times 10^{-2}$ & $(7.00 \pm 0.60) \times 10^{37}$ \\
\hline Swift XRT & 00031027001 & 7.3 & $2007-11-24.34$ & 125 & $(8.0 \pm 1.2) \times 10^{-3}$ & $(6.35 \pm 1.04) \times 10^{37}$ \\
\hline Chandra ACIS-S & 8187 & 5.0 & 2007-11-27.16 & 128 & $<6 \times 10^{-4}$ & $<9.7 \times 10^{35}$ \\
\hline Swift XRT & $00031027002 / 3$ & 4.7 & $2007-12-02.64$ & 133 & $(3.2 \pm 1.0) \times 10^{-3}$ & $(3.92 \pm 1.22) \times 10^{37}$ \\
\hline Swift XRT & 00031027004 & 3.9 & $2007-12-16.77$ & 147 & $<3.2 \times 10^{-3}$ & $<3.9 \times 10^{37}$ \\
\hline Swift XRT & 00031027005 & 4.0 & 2007-12-30.02 & 160 & $<2.0 \times 10^{-3}$ & $<2.4 \times 10^{37}$ \\
\hline XMM-Newton EPIC PN & 0511380201 & 22.8 & 2008-01-05.99 & 167 & $<5.1 \times 10^{-3}$ & $<3.5 \times 10^{36}$ \\
\hline
\end{tabular}

Notes: $\quad{ }^{a}$ Telescope and instrument used for observation. ACIS-S count rates are from Galache et al. (2007).

${ }^{b}$ Dead time corrected exposure time of the observation.

${ }^{c}$ Start date of the observation.

${ }^{d}$ Time in days after the last X-ray non-detection of SS2 on 2007 July 22.55 (JD = 2454304).

${ }^{e}$ Source count rates, X-ray luminosities (unabsorbed, blackbody fit, $0.2-1.0 \mathrm{keV}$ ) and upper limits were estimated according to Sect. 2.2.

with the XRT software task xrtexpomap within XIMAGE. Astrometry was done using the HEAsoft routine xrtcentroid.

For the XMM-Newton data we applied a background screening and used the XMMSAS v6.6 tasks eboxdetect and emldetect to detect sources in the image and perform astrometry and photometry. For computing upper limits we added an artificial detection at the position of the source to the eboxdetect list. This list was used as input for an emldetect run (with fixed positions and likelihood threshold of zero) that derived the observed flux and upper limit for all objects in the list. We give 3 times the background flux as the $3 \sigma$ upper limit. To obtain astrometrically-corrected positions we selected optical sources, with proper-motion corrected positions, from the USNO-B1.0 catalogue (Monet et al. 2003), and checked that only one optical source was in the error circle of the corresponding X-ray source. We only accepted sources correlating with globular clusters from 


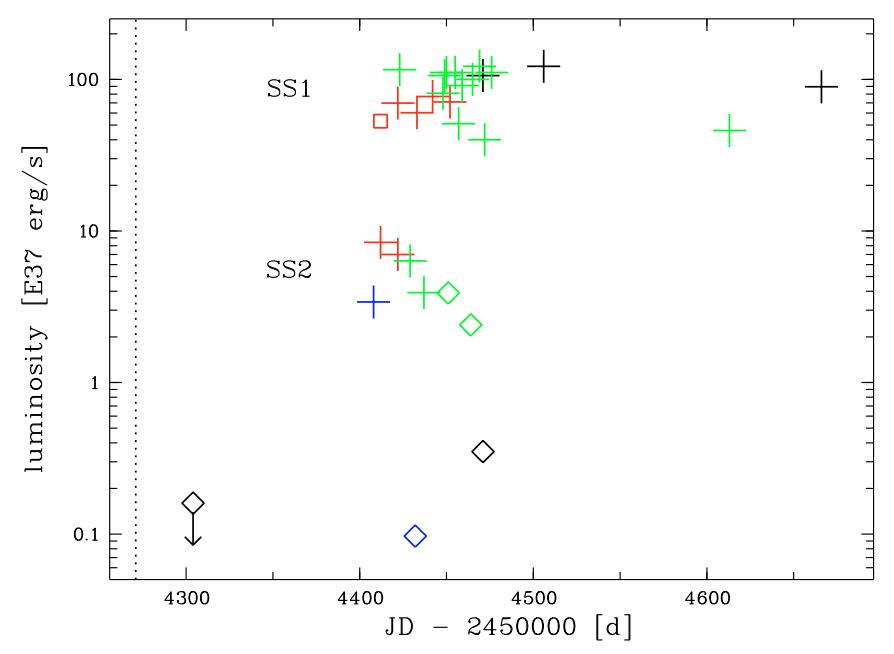

Fig. 1. X-ray light curves of SS1 and SS2 $(0.2-1.0 \mathrm{keV})$ obtained from XMM-Newton (black), Chandra HRC-I (red), Chandra ACIS-S (blue), and Swift XRT (green) (see also Tables 1,2). The upper limit for SS1 is indicated by a down-pointing arrow, the upper limits for SS2 are marked by open lozenges. An open square indicates the lower limit luminosity for SS1 on Chandra ObsID 8526. Crosses symbolise luminosities of detections. Error bars are not shown, since the (statistical) errors are mostly smaller than the size of the symbols. There are no errors given by Galache et al. (2007) for the ACIS-S observations. The vertical dotted line indicates the day of the first detection of nova M31N 2007-06b in the optical (Shafter \& Quimby 2007).

the Revised Bologna Catalogue (V.3.4, January 2008; Galleti et al. 2004, 2005, 2006, 2007) or with foreground stars, characterized by their optical to X-ray flux ratio (Maccacaro et al. 1988) and their hardness ratio (see Stiele et al. 2008). We then used the XMMSAS task eposcorr to derive the offset of the $\mathrm{X}$-ray aspect solution.

We reduced the Chandra observations with the CIAO v3.4 (Chandra Interactive Analysis of Observations) software package. The source detection was done with the CIAO tool wavedetect. Similar to the procedure described by Elvis et al. (2009) for the Chandra COSMOS survey, an adapted version of the XMMSAS tool emldetect was used to estimate background and exposure corrected fluxes and count rates for the detected sources. Both sources are located near the edge of the HRCI field of view and therefore both, photometry and astrometry, suffer from relatively large errors.

\subsection{Supersoft source in Bol 111}

In our first Chandra observation of the AO6 monitoring campaign, starting on 2007-11-07.64 UT (ObsID 8526), we detected a new source (SS1) at the very edge of the HRC-I field of view (Pietsch et al. 2007a). SS1 remained active during the following Chandra monitoring observations (ObsIDs 8527-30) in 2007 November and December. We followed the light curve of SS1 with Swift ToO observations (ObsIDs 0003101700112, 2007 November-2008 January) and also found the source to be still visible in our XMM-Newton M 31 monitoring observations (ObsIDs 0511380201 and 0511380601, 2008 January-February). In Table 1 we present details on all observations. Due to the location of SS1 near the edge of the Chandra HRC-I field of view during all observations (see Fig. 2 for the location of the Chandra field and the source), we used the XMM-Newton observations to perform precise astrometry.

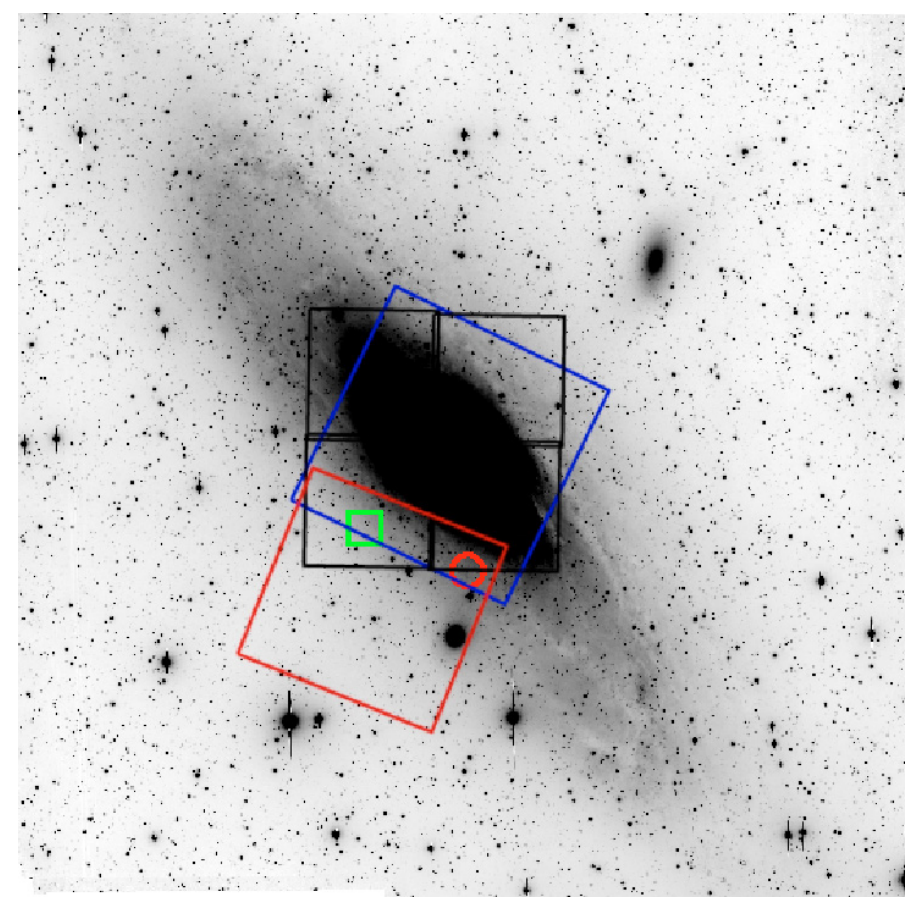

Fig. 2. ROTSE-III M 31 central field (see Sect.3). Overlaid are the four Super-LOTIS fields (black squares), the Chandra HRC-I field for ObsID 8527 (big blue square) and the XMM-Newton field for ObsID 0511380201 (big red square). Indicated are the position of Bol 111 (red circle) and Bol 194 (green square).

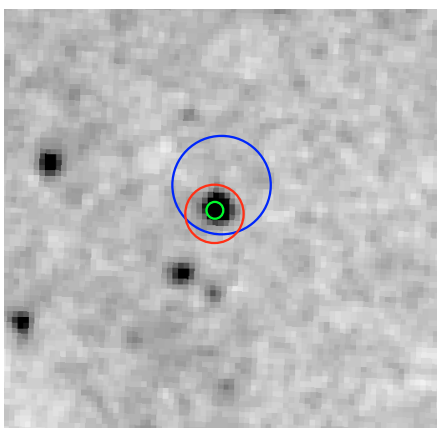

(a) Bol 111

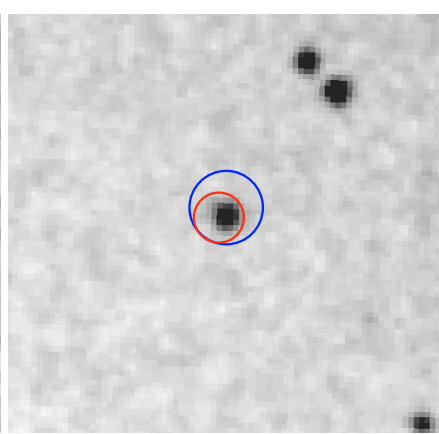

(b) Bol 194
Fig. 3. X-ray position error circles for SS1 and SS2 as described in Sects. 2.1 and 2.2 with respect to Bol 111 and Bol 194. Blue/big: Chandra HRC-I, red/medium: Swift XRT, green/small: XMMNewton PN. Underlying optical image: $5^{\prime} \times 5^{\prime}$ DSS POSS-II Red.

The offset-corrected XMM-Newton position of the source was determined to be RA(J2000) 00:42:33.21, Dec(J2000) $+41: 00: 26.1$ with a $3 \sigma$ error of $1^{\prime \prime} .6$, including the uncertainty of the offset correction. These coordinates are in good agreement (distance $=0$ '.5), within the errors, with the position of the M 31 GC Bol 111 (Galleti et al. 2004, 00:42:33.16, +41:00:26.1). Therefore, we assume that SS1 is situated within the globular cluster. Note, that no X-ray source was previously known in this GC. See Fig. 3 for a visualisation of the agreement of optical (DSS POSS-II Red) and X-ray positions.

To perform spectral analysis of SS1 we used XMMNewton observations obtained on 2008-01-05.99 UT and 200802-09.31 UT (ObsIDs 0511380201 and 0511380601). We extracted the spectra of SS1 from both observations and fitted them simultaneously in order to increase statistics. We used data from 


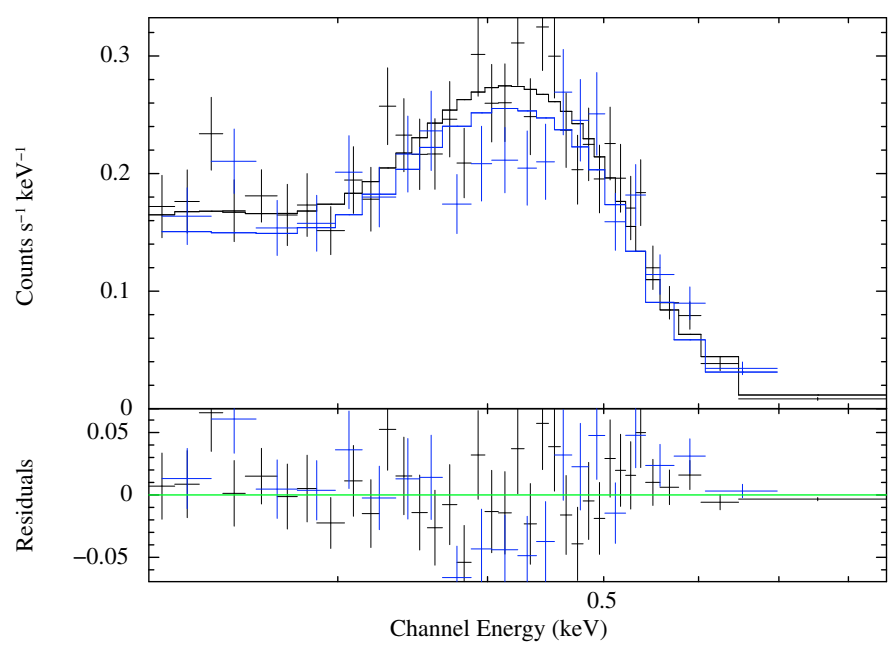

Fig. 4. XMM-Newton EPIC PN spectra of SS1 (crosses) from observations 0511380201 (black) and 0511380601 (blue) fitted with an absorbed blackbody (solid lines).

XMM-Newton's PN detector because of the better sensitivity of PN in the soft band compared to both MOS detectors. The spectra were extracted using XMMSAS task evselect. For both spectra only single-pixel events (PATTERN $=0$ ) were selected.

We fitted the spectra in XSPEC using an absorbed blackbody approach. Temperature and foreground $N_{\mathrm{H}}$ were both assumed to be the same during the two observations and only the respective normalisations were allowed to vary independently from each other. The relative stability of the spectral parameters is confirmed by a XMM-Newton observation on 2008-0718.26 UT (160 days later than 051138601), from which we extracted a spectrum of SS1 that can be fitted by a model with similar parameters (see below). The blackbody approach yields an acceptable $\chi_{r}^{2}=1.39$ for the best fit values of $k T=48_{-3}^{+2} \mathrm{eV}$ and $N_{\mathrm{H}}=2.3 \pm 0.1 \times 10^{21} \mathrm{~cm}^{-2}$. This fit is shown in Fig. 4 and the associated contour plot is given in Fig. 5. We computed the unabsorbed EPIC PN X-ray luminosities, in the range $0.2-1.0 \mathrm{keV}$, from the best fit model in XSPEC and used the best fit values to create in XSPEC fake spectra (command fakeit) to infer the energy conversion factors (ecf) for the Swift XRT $\left(\right.$ ecf $\left.f_{\text {XRT }}\right)$ and the Chandra HRC-I (ecf $\left.f_{\text {HRC-I }}\right)$. The ecf values are given in Table 3 and were used to convert our Swift XRT and Chandra HRC-I count rates to unabsorbed luminosities. All luminosities assume a distance to M 31 of $780 \mathrm{kpc}$ (Holland 1998; Stanek \& Garnavich 1998) and are presented in Table 1. Note, that for our first detection of SS1 in the Chandra HRC-I observation 8526 no photometry is possible due to the location of the source on the edge of the detector. The blackbody fit parameters and derived values, like luminosities for ObsID 0511380201, are given in Table 3 .

The unabsorbed X-ray luminosities inferred from the blackbody fit are in the order of $10^{39} \mathrm{erg} \mathrm{s}^{-1}$, and therefore significantly exceed the Eddington luminosity of the hydrogen rich atmosphere of a WD: $L_{\text {Edd }}=1.3 \times 10^{38}\left(\frac{M}{M_{\odot}}\right) \mathrm{erg} \mathrm{s}^{-1}$. The fact that blackbody fits to SSS spectra produce in general too high values of $N_{\mathrm{H}}$ and too low temperatures, and therefore too high luminosities, is well known (see e.g. Greiner et al. 1991; Kahabka \& van den Heuvel 1997, and references therein), thus the values given in Table 1 define upper limits on the actual luminosity of SS1.

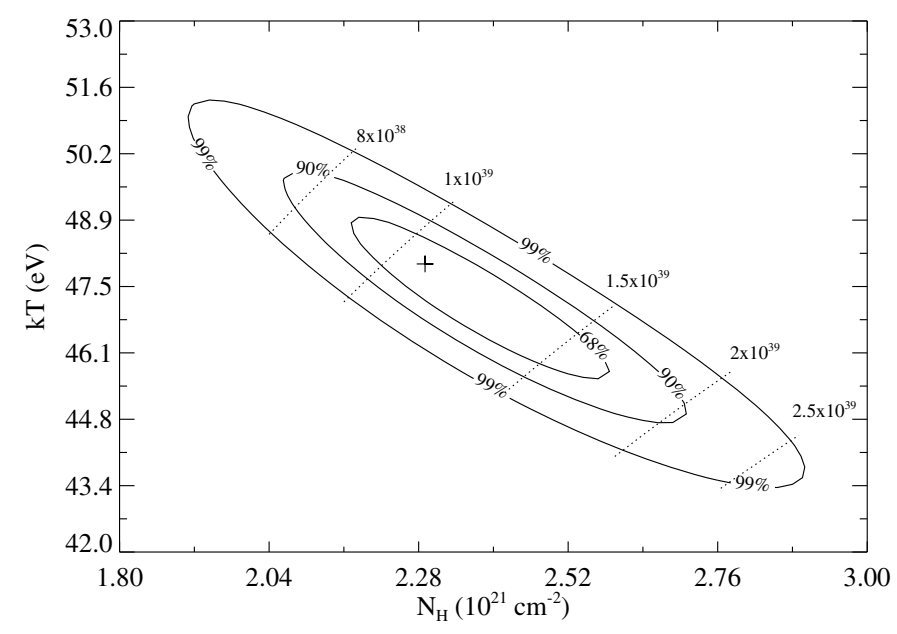

Fig. 5. Column density $\left(N_{\mathrm{H}}\right)$ - temperature $(k T)$ contours inferred from the blackbody fit to the XMM-Newton EPIC PN spectra of SS1 (see Fig. 4). Normalisation has been adjusted. Indicated are the formal best fit parameters (cross) and the lines of constant X-ray luminosity $(0.2-$ $1.0 \mathrm{keV}$, dotted lines).

Table 3. Comparison of SS1 spectral best fit parameters and derived parameters for blackbody and WD atmosphere models with halo and solar abundances.

\begin{tabular}{|c|c|c|c|}
\hline $\begin{array}{l}\text { Model } \\
\text { (energy range }[\mathrm{keV}])\end{array}$ & $\begin{array}{r}\text { Blackbody } \\
(0.2-0.8)\end{array}$ & $\begin{array}{l}\text { WD halo } \\
(0.2-0.6)\end{array}$ & $\begin{array}{l}\text { WD solar } \\
(0.2-0.6)\end{array}$ \\
\hline $\mathrm{kT}(\mathrm{eV})$ & $48_{-3}^{+2}$ & $61 \pm 1$ & $70 \pm 1$ \\
\hline$N_{\mathrm{H}}\left(10^{21} \mathrm{~cm}^{-2}\right)$ & $2.3 \pm 0.1$ & $1.0 \pm 0.2$ & $1.0 \pm 0.2$ \\
\hline$\chi_{r}^{2}$ & 1.39 & 1.01 & 1.06 \\
\hline d.o.f. & 53 & 44 & 48 \\
\hline$L_{x}\left(10^{38} \mathrm{erg} \mathrm{s}^{-1}\right)$ & $10.6 \pm 0.2$ & $1.00 \pm 0.02$ & $0.88 \pm 0.02$ \\
\hline$L_{b o l}\left(10^{38} \mathrm{erg} \mathrm{s}^{-1}\right)$ & $28.7_{-0.1}^{+0.2}$ & $2.7 \pm 0.1$ & $1.5 \pm 0.1$ \\
\hline$R\left(10^{9} \mathrm{~cm}\right)$ & $7.0_{-07}^{+1.6}$ & $1.21 \pm 0.07$ & $0.70 \pm 0.04$ \\
\hline $\operatorname{ecf}_{\mathrm{PN}}\left(\mathrm{ct} \mathrm{cm}^{2} \mathrm{erg}^{-1}\right)$ & $5.0 \times 10^{9}$ & $5.5 \times 10^{10}$ & $6.1 \times 10^{10}$ \\
\hline $\operatorname{ecf}_{\mathrm{HRC}-\mathrm{I}}\left(\mathrm{ct} \mathrm{cm}^{2} \mathrm{erg}^{-1}\right)$ & $2.6 \times 10^{9}$ & $3.2 \times 10^{10}$ & $3.7 \times 10^{10}$ \\
\hline $\mathrm{ecf}_{\mathrm{XRT}}\left(\mathrm{ct} \mathrm{cm}^{2} \mathrm{erg}^{-1}\right)$ & $7.4 \times 10^{8}$ & $8.7 \times 10^{9}$ & $9.9 \times 10^{9}$ \\
\hline
\end{tabular}

Notes: Luminosities and WD radii refer to the XMM-Newton observation 0511380201 . The unabsorbed X-ray luminosity $L_{x}$ is for the $0.2-1.0 \mathrm{keV}$ range. Bolometric luminosities and WD radii for the WD atmosphere model are upper limits (see Sect.2.1 for details).

Blackbody fits are very simple approximations for SSS emission of novae and allow to compare parameters like effective temperature for different novae. However, as mentioned above, these fits are physically not realistic. Therefore, we tested fitting our low-resolution spectra with WD atmosphere models, that are based on more physical assumptions. We used a grid of synthetic ionizing spectra for hot compact stars from NLTE model atmospheres computed by Rauch (2003). These NLTE models are plane-parallel, in hydrostatic and radiative equilibrium and contain all elements from $\mathrm{H}$ to $\mathrm{Ca}$ (Rauch 1997). The models were computed using the Tübingen Model-Atmosphere Package (TMAP, Rauch \& Deetjen 2003). Elemental abundances are fixed to either galactic halo $\left([X]^{3}=[Y]=0,[Z]=-1\right)$ or so$\operatorname{lar}([X]=[Y]=[Z]=0)$ ratios. The grids of model atmosphere fluxes, as well as FITS tables which can be used in XSPEC, are

\footnotetext{
${ }^{3}$ []: $\log$ (abundance/solar abundance).
} 


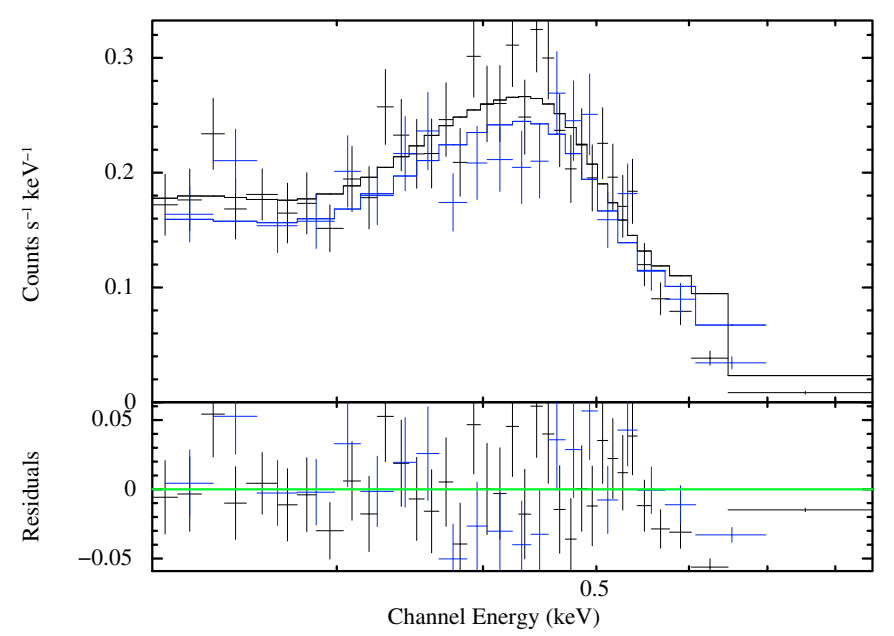

Fig. 6. XMM-Newton EPIC PN spectra of SS1 from observations 0511380201 (black) and 0511380601 (blue) fitted with an WD atmosphere model with galactic halo abundances (solid lines).

available on-line ${ }^{4}$. The XSPEC tables contain temperatures and fluxes (binned to $0.1 \AA$ intervals) for fixed surface gravity $(\log g$ ) and elemental abundances. In our case, the available grid parameter space was restricted to models with $\log g=9.0$, since only these tables include temperatures high enough to fit our spectra. This restriction clearly limits the significance of our best fits, since models with different surface gravity may have provided equally good fits with different parameters. Also note, that the assumptions of plane parallel and static are not physically realistic for a nova atmosphere. Furthermore, the spectral analysis is limited by the low energy resolution of the EPIC PN spectra, which are, due to the faintness of the source, the only available spectra with sufficient signal to noise ratio. Great caution should therefore be applied when interpreting the results of our test fits.

We fitted the EPIC PN spectra with both halo and solar abundance ratios. Galactic halo abundances are more representative of the metallicity of the M 31 GCs (Barmby \& Huchra 2000). Figure 6 gives the fit for halo abundances and shows that the atmosphere model fits the spectrum well only up to an energy of about $600 \mathrm{eV}\left(\chi^{2}\right.$ d.o.f. $\left.{ }^{-1}=1.01\right)$ but predicts too high flux at energies above, which is clearly indicated by the residuals. This is true for solar and halo abundances. However, this is no surprise since recent detailed X-ray spectroscopy of the SSS phase of galactic novae shows that these spectra are complex (see e.g. Ness et al. 2007, who used spectra obtained with the XMM-Newton Reflection Grating Spectrometer (RGS) and the Chandra Low Energy Transmission Gratings Spectrometer (LETGS)). Spectral models currently available may be not suitable for a correct fitting of SSS spectra of novae, especially with respect to the elemental abundances of a hot post-nova atmosphere.

In Table 3 we compare the parameters of the WD atmosphere model fits (fitted to the $0.2-0.6 \mathrm{keV}$ range) to the parameters of the blackbody fit described above. We give unabsorbed X-ray luminosities $\left(L_{x}\right)$, that were computed for the range $0.2-1.0 \mathrm{keV}$ in XSPEC, and bolometric luminosities $\left(L_{\mathrm{bol}}\right)$ as well as WD radii $(R)$, inferred from the bolometric luminosities. The bolometric luminosity for the blackbody fit was directly computed from the normalisation of the spectra in XSPEC. For the WD atmosphere model we used an absorbed blackbody fit

${ }^{4}$ http://astro.uni-tuebingen.de/ rauch/ with the same temperature and foreground $N_{\mathrm{H}}$ to compute bolometric luminosity and WD radius. These values overestimate the actual luminosity and radius and are therefore upper limits. Energy conversion factors (ecf) for the Swift XRT $\left(\operatorname{ecf}_{X R T}\right)$ and the Chandra HRC-I ( ecf $\left._{\mathrm{HRC}-\mathrm{I}}\right)$ detectors were again computed in XSPEC, using fake spectra as for the blackbody fit, and are listed in Table 3 together with the ecf for the XMM-Newton EPIC PN $\left(e f_{\mathrm{PN}}\right)$.

The comparison of the fits shows that the WD atmosphere models give physically more plausible results. The high value of $N_{\mathrm{H}}$ which was required for the blackbody fit (three times the foreground absorption) is significantly reduced to a value which is just slightly above the foreground level of $N_{\mathrm{H}}=$ $0.7 \times 10^{21} \mathrm{~cm}^{-2}$. As a consequence, the super-Eddington luminosity derived from the blackbody fits is reduced to more realistic values for the atmosphere models. The connection of $N_{\mathrm{H}}$ and effective temperature can be seen in the confidence contours shown in Fig. 5. The effective temperature for the WD atmosphere is higher than the blackbody temperature and is revealing the H-burning hot layer. Still, one has to be cautious interpreting these models, because the elemental abundances are fixed and may be not realistic to describe abundances in a burning WD atmosphere. However, the consideration of model substructures, like absorption edges, already causes a reduction of the $N_{\mathrm{H}}$ necessary to adjust the model to the spectra. Truly physical models for supersoft emission from novae are strongly needed and should be validated using high resolution spectra obtained with current and future missions.

We checked recent Swift (ObsID 00037718001, starting at 2008-05-26.29 UT) and XMM-Newton (ToO 0560180101, starting at 2008-07-18.26 UT) data and found that SS1 is still visible in both observations (see also Table 1 for details). Preliminary data analysis was published in Pietsch et al. (2008a,b). For Swift we used the blackbody fit described above and in Table 3 to convert the XRT count rate to unabsorbed luminosity. For XMM-Newton we extracted an EPIC PN spectrum of SS1 as described above for the two earlier XMM-Newton observations. The spectrum can be fitted with an blackbody fit with best fit values of $k T=43 \pm 8 \mathrm{eV}$ and $N_{\mathrm{H}}=2.2_{-0.7}^{+1.0} \times 10^{21} \mathrm{~cm}^{-2}\left(\chi_{r}^{2}=1.52\right)$. These parameters are consistent with the blackbody fit described above and result in an unabsorbed X-ray luminosity (range of $0.2-1.0 \mathrm{keV})$ of $(8.9 \pm 0.5) \times 10^{38} \mathrm{erg} \mathrm{s}^{-1}$. Both observations show that the X-ray luminosity of SS1 is slowly declining.

Supersoft spectra like the one of SS1 are typical for counterparts to optical novae and indicate that after the outburst there is hydrogen burning going on in the remaining envelope of the WD (see Pietsch et al. 2005a, and references therein). Therefore, we identify the supersoft X-ray transient in the globular cluster Bol 111 with the nova M31N 2007-06b reported by Shafter \& Quimby (2007). This optical nova was first detected on 2007 June 19.38 and was detected in X-rays the first time 141 days later on 2007 November 07.64. SS1 was not visible on 2007 July 22.55 , which is 33 days after the first detection in the optical (see Table 1).

\subsection{Supersoft source in Bol 194}

A second supersoft source (SS2) was detected in the same Chandra HRC-I observation as SS1 (ObsID 8526) as a new, bright X-ray transient (Haberl et al. 2007a). In the following observation (ObsID 8527) SS2 was again detected with similar brightness close to the edge of the HRC-I field of view (see Fig. 2). For the remaining three Chandra observations of the monitoring campaign the position of SS2 was outside the 


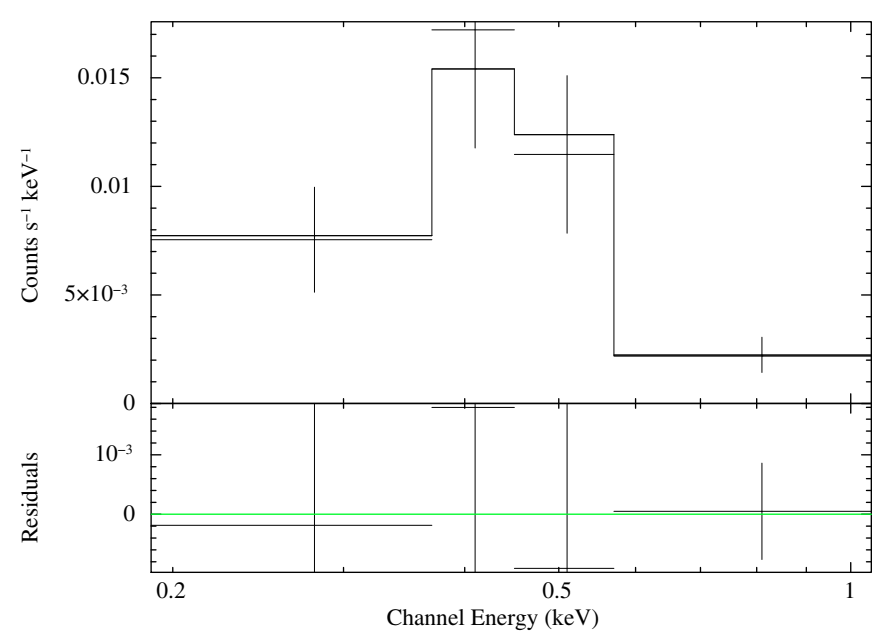

Fig. 7. Swift XRT spectrum of SS2 in Bol 194 from observation 00031027001 (7.3 ks exposure time) fitted with an absorbed blackbody fit (solid line).

field of view due to the changing roll angle of the observations. Therefore, we used our Swift XRT ToO follow up observations (starting with ObsID 00031027001) to constrain the spectrum of SS2 and follow the X-ray light curve. The X-ray luminosities given in Table 2 show that this source faded much faster than SS1. Therefore, no XMM-Newton observations of SS2 are available and we could use our XMM-Newton data only to compute upper limits. Since SS2 also has a large off-axis position in the HRC-I field, we used the Swift observation 00031027001 to compute the position of the source. We found the following coordinates: RA(J2000) 00:43:45.3, Dec(J2000) +41:06:08.15. With a $1 \sigma$ position error of 4 ". 6 and a distance of 1 ". 1 to the GC Bol 194 (Galleti et al. 2004, 00:42:45.20, +41:06:08.3) these coordinates are in good agreement with the position of Bol 194. See Fig. 3 for a comparison of optical (DSS POSS-II Red) and X-ray positions.

The Swift XRT spectrum of SS2 (ObsID 00031027001) only shows photons with energies below $750 \mathrm{eV}$ (see Fig. 7). Therefore, we classified this source as a SSS. Since we have not many spectral counts for this source, we use Cash statistics for spectral modelling. The best spectral fit is an absorbed blackbody with best fit values of $k T=74_{-23}^{+32} \mathrm{eV}$ and $N_{\mathrm{H}}=$ $\left(1.0_{-0.9}^{+1.6}\right) \times 10^{21} \mathrm{~cm}^{-2}$. The best fit parameters differ from the preliminary data analysis published in Haberl et al. (2007b), where we used a different abundance table in XSPEC (angr; Anders \& Grevesse 1989) and $\chi^{2}$ statistics. Using the best fit values we computed the X-ray luminosity of SS2 to be $(6.4 \pm 1.0) \times 10^{37} \mathrm{erg} \mathrm{s}^{-1}$, the bolometric luminosity to be $10_{-4}^{+7} \times 10^{37} \mathrm{erg} \mathrm{s}^{-1}$ and therefore the radius of the WD to be $5_{-3}^{+11} \times 10^{8} \mathrm{~cm}$. Unfortunately, the low count $S$ wift XRT spectrum does not provide enough degrees of freedom to test the WD atmosphere model that we fitted to the XMM-Newton EPIC PN spectra of SS1.

After our discovery, Galache et al. (2007) reported that they found SS2 to be present in a $5 \mathrm{ks}$ Chandra ACIS-S observation taken on 2007-11-03.18 UT, which is four days earlier than our first Chandra HRC-I detection. They confirm our classification of SS2 as a SSS and further report that the source is not visible anymore in a $5 \mathrm{ks}$ Chandra ACIS-S observation on 200711-27.16 UT ( $\sim 24$ days after the previous ACIS observation) with an $95 \%$ upper detection limit of $\sim 6 \times 10^{-4} \mathrm{ct} \mathrm{s}^{-1}$. Note, that this observation was taken just three days after our Swift observation on 2007-11-24.34 UT. Additional Swift XRT observations by Kong \& Di Stefano (2007a) showed a re-brightening of the source on 2007-12-02.64. However, the source faded quickly again and was not found in follow-up Swift observations and our XMM-Newton monitoring data. We re-analysed the Swift and Chandra HRC-I data, computed upper limits for the XMM-Newton observations and present all available data on SS2 in Table 2. We took the count rates for detections and upper limits from Kong \& Di Stefano (2007a) to compute luminosities in XSPEC using our spectral model. For the Swift observations $00031027004 / 5$ we give $95 \%$ upper limits on the SS2 luminosity. These values are comparable to actual luminosities obtained for earlier Swift observations, an effect which is due to longer effective source exposure times for the earlier observations. X-ray luminosities for the three instruments were computed using XSPEC and the blackbody fit inferred from the Swift spectrum.

Analogous to SS1 and its possible connection to M31N 2007-06b in Bol 111, the supersoft spectrum and the transient light curve could indicate that SS2 is the X-ray counterpart of a recent optical nova in the GC Bol 194. The following section describes the optical data analysis and the constraints that we can put on a possible counterpart nova in Bol 194.

\section{Search for an optical nova counterpart of the supersoft source in Bol 194}

As there was no optical nova reported in Bol 194 we searched the recent optical data available to us for indications of a nova outburst in the GC. Note, that the constant background light from the GC makes it difficult to detect nova outbursts. However, difference imaging is a successful method in this context and was used to analyse a big part of our optical data set. In the following we describe the observational setup and the analysis of the optical data.

\subsection{Optical observations}

The majority of our optical data was obtained in the context of the Texas Supernova Search (TSS; Quimby 2006). The TSS employed the 0.45-m ROTSE-IIIb telescope at the McDonald observatory in Texas, and the data presented here are supplemented by its twin, the ROTSE-IIId telescope, which is located at the Turkish National Observatory at Bakirlitepe, Turkey. The ROTSE-III system is described in Akerlof et al. (2003). The $1.85 \times 1.85$ field of view covered by the unfiltered, $2 \mathrm{k} \times 2 \mathrm{k}$ Marconi CCD encompasses practically all of M 31's light in a single exposure (see Fig. 2), and additional overlapping fields to the northeast and southwest add to the haul. Beginning in November 2004, these 3 fields were imaged several times nightly as weather and season allowed.

Our second monitoring program for optical novae uses the robotic $60 \mathrm{~cm}$ telescope with an E2V CCD $(2 \mathrm{k} \times 2 \mathrm{k})$ Livermore Optical Transient Imaging System (Super-LOTIS, Williams et al. 2008) located at Steward Observatory, Kitt Peak, Arizona, USA. Starting in October 2007 the telescope was used in every good night to monitor the bulge of M31. Using four SuperLOTIS fields (field of view: $17^{\prime} \times 17^{\prime}$ ) these observations cover an area of $\sim 34^{\prime} \times 34^{\prime}$ centered on the core of M31 with a pixel scale of $0.496^{\prime \prime} /$ pixel and a typical limiting magnitude of $19.0 \mathrm{mag}$, using a Johnson $R$ filter. The reduction of the data 
is done by a semi-automatic routine and the astrometric and photometric calibration uses the M 31 part of the Local Group Survey (LGS, Massey et al. 2006). Typical values of SuperLOTIS astrometric and photometric $1 \sigma$ accuracies are 0 ".25 and $0.25 \mathrm{mag}$, respectively, averaged over the whole magnitude range. The southeast Super-LOTIS field covers Bol 194 and the astrometric and photometric $1 \sigma$ accuracies for this particular object ( 16.8 mag) are 0 ". 11 and $0.06 \mathrm{mag}$, respectively.

Additionally, in this research our optical data set is supplemented by archival data from K. Hornoch obtained at telescopes in Lelekovice (Newtonian focus of 350/1658 mm telescope, CCD camera G2CCD-1600, Kron-Cousins $R$ filter, $1.12^{\prime \prime} /$ pixel, FOV 28.7'x19.1') and Ondřejov (primary focus of 650/2342 mm telescope, CCD camera G2CCD-3200, Kron-Cousins $R$ filter, $1.20^{\prime \prime} /$ pixel, FOV $\left.21.8^{\prime} \times 14.7^{\prime}\right)$. Standard reduction procedures for raw CCD images were applied (dark and bias subtraction and flat-field correction) using SIMS $^{5}$ and Munipack 6 programs. Reduced images of the same series were co-added to improve the $\mathrm{S} / \mathrm{N}$ ratio (total exposure time varied from 600 s up to 1800 s). The gradient of the galaxy background of co-added images was flattened by the spatial median filter using SIMS. These processed images were used for aperture photometry, carried out in GAIA ${ }^{7}$. Relative photometry was done using brighter field stars which were calibrated using standard Landolt fields. The $1 \sigma$ measurement uncertainties were low ( $\sim 0.03 \mathrm{mag})$, thanks to the long exposure times, the brightness of Bol 194, and its location far from the high surface brightness levels found near the center of M 31 .

\subsection{Optical data analysis}

We searched the optical data of Bol 194 obtained by the monitoring programs described above for a significant optical excess that could indicate a nova outburst in the GC. The significance for the Super-LOTIS data and for Hornoch's data is defined by applying a $3 \sigma$ clipping to the light curves of Bol 194 and judging every two neighboured data points that lie outside the final $\pm 3 \sigma$ range as possible indications of a nova outburst.

For the ROTSE-III data, we used the PSF-matched image subtraction code developed by the Supernova Cosmology Project (Perlmutter et al. 1999) to search for residual light from an optical nova. With this method the outburst of nova M31N 2007-06b in Bol 111 was detected (Shafter \& Quimby 2007). We first constructed a deep reference image by co-adding 100 ROTSE-IIIb images obtained between December 2004 and June 2006. We then convolved this image to match the PSF of each ROTSE-III image, subtracted off this template light, and searched for any point sources coincident with Bol 194. We attempted to measure any residuals at this location with the DAOPHOT PSF-fitting routines (Stetson 1987; ported to IDL by Landsman 1989).

These procedures did not lead to the discovery of an optical nova counterpart of SS2 in Bol 194. However, our optical monitoring data of the M 31 bulge region allows us to put strong constraints on the outburst date of any nova that may have occurred in Bol 194. Figure 8 includes all our optical data of Bol 194 from November 2004 until 2007-11-08, which is 5 days after the first detection of SS2 in X-rays on 2007-11-03. We show the minimum detectable magnitude of a possible optical nova occurring in Bol 194 during this period.

\footnotetext{
5 http://ccd.mii.cz/

6 http://munipack.astronomy.cz/

7 http://wWW. starlink.rl.ac.uk/gaia
}

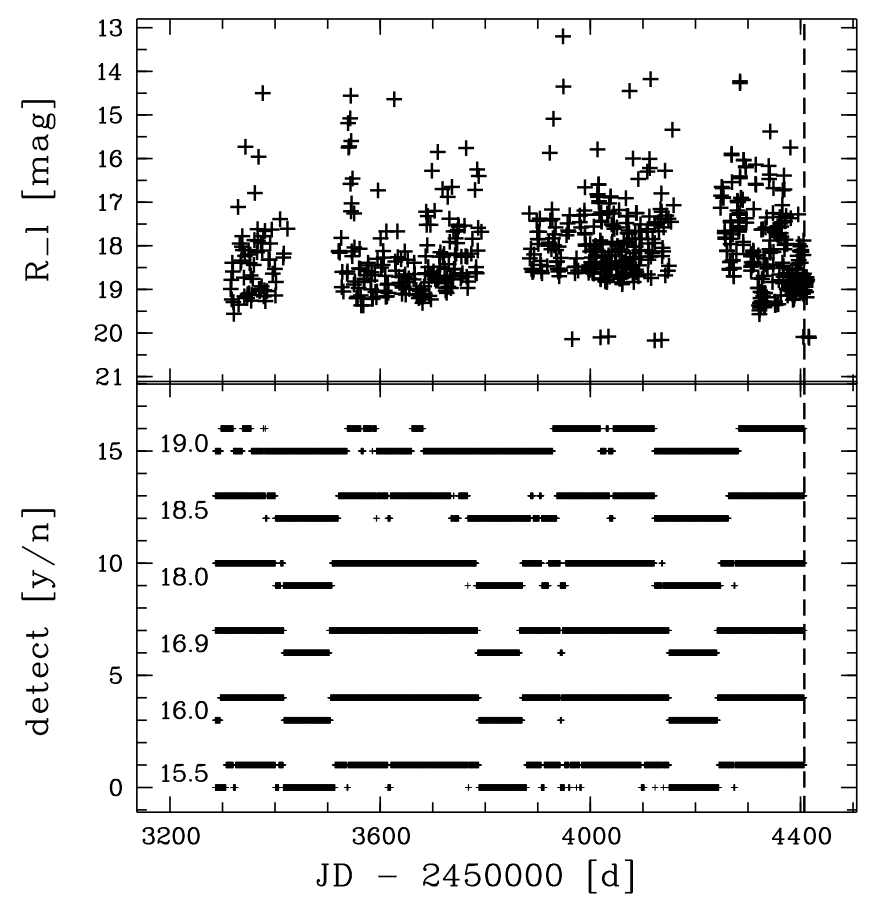

Fig. 8. Top: Limiting magnitudes of all optical data since 2004 November. Bottom: simulated detection (upper points) or non detection (lower points) for novae with indicated peak magnitudes. In both panels the vertical dashed line shows the date of the first detection of SS2 in X-rays.

To calculate the limiting magnitudes of the ROTSE-III observations, we measured the noise on the subtracted frames in annuli centered on the location of Bol 194. The limits reported here correspond to the flux required of a point source to be detected at the $4 \sigma$ level. The magnitude scale was calibrated against the USNO-B1.0 R2 measurements (Monet et al. 2003).

In contrast to this procedure, the limiting magnitude for the Super-LOTIS data and for Hornoch's data is computed as follows. At the distance of M 31 the light of a nova in a GC would blend with the light of the GC itself. Therefore, we have to take into account the intrinsic magnitude of the cluster $(\sim 16.8 \mathrm{mag}$ in the Super-LOTIS data) and compute the resulting magnitude using

$R_{\mathrm{t}}=-2.5 \log _{10}\left(10^{-0.4 R_{\mathrm{gc}}}+10^{-0.4 R_{\mathrm{n}}}\right)$,

with $R_{\mathrm{t}}, R_{\mathrm{gc}}$, and $R_{\mathrm{n}}$ being the total magnitude, the magnitude of the GC, and the magnitude of the nova, respectively. Thus, for the Super-LOTIS data and for Hornoch's data, the minimum detectable magnitude $R_{1}$ of a nova is defined as the magnitude that leads to a significantly brighter $R_{\mathrm{t}}$. The significance criterion used here is:

$R_{\mathrm{t}}<R_{\mathrm{gc}}-3 \sigma_{R_{\mathrm{gc}}}$,

with $3 \sigma_{R_{\mathrm{gc}}}$ being the $3 \sigma$ photometric standard error for Bol 194 ( $~ 0.16$ mag for the Super-LOTIS data reduction and $\sim 0.09 \mathrm{mag}$ for Hornoch's data). These measurement errors are mean values derived from our light curves of Bol 194. Since we used a $3 \sigma$ clipping method to search for a nova outburst in Bol 194, we assume that the mean photometric errors given for both instruments would correspond to the final $3 \sigma$ range in the case of a nova outburst-modified light curve. Therefore, we compute $R_{1}$ as follows:

$R_{1}=-2.5 \log _{10}\left(10^{-0.4\left(R_{\mathrm{gc}}-3 \sigma_{R \mathrm{gc}}\right)}-10^{-0.4 R_{\mathrm{gc}}}\right)$. 
In order to judge the time coverage of our data set, we simulated the outburst of novae with different peak magnitudes and decay times in Bol 194 on any day between 2004 October and 2007 November and checked whether we would have detected the outburst according to the limiting magnitudes, or not. We simulated novae with peak magnitudes of $15.5,16.0,16.9,18.0$, 18.5 and $19.0 \mathrm{mag}$ in the $R$ band and decay times of 6,15 , 29, 63, 102 and 164 days, respectively. The peak magnitude of nova M31N 2007-06b in Bol 111 as given in Shafter \& Quimby (2007) is 16.9 mag. The decay time $t_{2}$ is defined as the time in days the nova luminosity needs to drop 2 mag below peak luminosity (Payne-Gaposchkin 1964). To compute this value, we used the maximum magnitude versus rate of decline (MMRD) relationship given by Della Valle \& Livio (1995), for $t_{2} \leq 50 \mathrm{~d}$ :

$M_{V, \max }=-7.92-0.81 \arctan \left(\frac{1.32-\log t_{2}}{0.23}\right)$,

and by Cohen (1988), for $t_{2}>50 \mathrm{~d}$ :

$M_{V, \max }=2.41 \log t_{2}-10.70$.

We used this combination of the two different MMRDs according to Shafter (1997) in order to compensate for the breakdown of the MMRD from Della Valle \& Livio (1995) at large values of $t_{2}$. Both MMRDs give the relation of the decay time of a nova to its maximum magnitude in the $V$ band. The color relation for novae at maximum light is $(B-V)_{0}=0.23 \pm 0.06$ (van den Bergh $\&$ Younger 1987). This fact implies for novae at maximum light a spectral type close to $\mathrm{F} 0$ and therefore a $(V-R)_{0} \sim 0.30$ (see e.g. Allen 1976) which we used to match both MMRDs to our $R$ band data.

For our simulations we used a M 31 extinction free distance modulus of $\mu_{0}=24.38 \mathrm{mag}$ (Freedman et al. 2001). We applied a reddening towards M 31 of $E(B-V)=0.062$ (Schlegel et al. 1998) and a foreground extinction in the $R$ band of $A_{R}^{\mathrm{f}}=0.17 \mathrm{mag}$, obtained via the NASA Extragalactic Database (NED). We estimated the internal absorption of M 31 to be $A_{R}^{\mathrm{i}} \sim 0.11 \mathrm{mag}$, using $A_{p g}^{\mathrm{i}} \sim 0.20 \mathrm{mag}$ (Capaccioli et al. 1989) and assuming that $A_{p g} \sim A_{B}$.

The results of these simulations are shown in the top panel of Fig. 8, with the upper parts of the curves indicating the periods over which each simulated nova would have been detected. The figure visualises the fact that our monitoring is efficient in the sense that the only periods in which we would not have been able to detect the most novae, are the periods when M 31 is not observable due to its annual visibility. These periods, computed for a nova with parameters of M31N 2007-06b, and the estimated constraints on the delay time between optical outburst and detection in X-rays are shown in Table 4. Note also, that a bright nova (peak luminosity $=15.5 \mathrm{mag}$ ) would have been missed more likely than a fainter nova. This is due to the faster decline of luminosity for the brighter novae and good detection limits of our observations down to $\sim 19 \mathrm{mag}$.

\section{Discussion}

\subsection{Summary of observational data}

We present in this paper the first two SSSs found in M 31 GCs. The source SS1 in Bol 111 can be identified with M31N 200706b, the very first nova found in a M 31 GC in June 2007 (Shafter \& Quimby 2007). After 137 days (November 2007) we detected supersoft X-ray emission from this source and it was still active in our last XMM-Newton observation in July 2008.
Table 4. Optical constraints on a nova in Bol 194.

\begin{tabular}{lccll}
\hline \hline Date1 [UT] $^{a}$ & Date2 [UT] $^{a}$ & RJD1 [d] $^{b}$ & RJD2 [d] $^{b}$ & Delay [d] $^{c}$ \\
\hline $2005-02-15$ & $2005-05-12$ & 3417 & 3503 & $990-904$ \\
$2006-02-19$ & $2006-05-09$ & 3786 & 3865 & $621-542$ \\
$2007-02-18$ & $2007-05-20$ & 4150 & 4241 & $257-166$ \\
\hline
\end{tabular}

Notes: ${ }^{a}$ Start date (date1) and end date (date2) of the nondetection periods.

${ }^{b}$ The same as in ${ }^{a}$ but in RJD $=\mathrm{JD}-2450000$.

${ }^{c}$ Periods of possible delay time between nova outburst and first detection in X-rays.

The supersoft spectrum and the transient nature of SS2 in Bol 194 could also indicate a recent nova outburst. However, we could not find evidence for an nova counterpart in optical monitoring data of this GC. We give constraints on a possible nova outburst in Bol 194, based on the detection limits of our optical observations and simulated nova outbursts. The simulations show that most novae would have been detected in Bol 194, except in the times when M 31 could not be observed due to it's annual visibility. We computed the time spans in which a nova like M31N 2007-06b in Bol 194 could have had an unobserved outburst. The associated delay times, given in Table 4, between optical outburst and X-ray switch-on are physically feasible, since similar delays have been observed in other supersoft nova counterparts (see Pietsch et al. 2007b). Therefore, a nova outburst in Bol 194, that is connected to SS2, cannot be ruled out.

\subsection{Globular cluster nova rate - the optical point of view}

The statistics of novae in GCs of other galaxies is still poor. Tomaney et al. (1992) conducted a search in $\mathrm{H} \alpha$ of over 200 M 31 GCs for nova eruptions and found nothing over an effective survey time of one year. This results in an upper limit for the M 31 GC nova rate of 0.005 novae $\mathrm{yr}^{-1} \mathrm{GC}^{-1}$. Another search for novae in 54 M 31 GCs was done by Ciardullo et al. (1990b) based on the M $31 \mathrm{H} \alpha$ survey data of Ciardullo et al. (1987, 1990a). Over a mean effective survey time of approximately two years no indications for a nova outburst in one of the GCs was found. From one nova found in a GC of the giant elliptical galaxy M 87 Shara et al. (2004) derive a rate of 0.004 novae $\mathrm{yr}^{-1} \mathrm{GC}^{-1}$ for the whole system of 1057 known M 87 GCs $\left(4.2\right.$ novae $\left.\mathrm{yr}^{-1}\right)$.

There are about 500 GCs known that belong to M 31 (482 confirmed GCs, according to the Revised Bologna Catalogue ${ }^{8}$ (V.3.5, March 2008; Galleti et al. 2004, 2005, 2006, 2007)). This corresponds approximately to a stellar mass of about $5 \times 10^{9} M_{\odot}$. Given a rate of 2.2 novae $\mathrm{yr}^{-1}\left(10^{10} M_{\odot}\right)^{-1}$, which is observed in old systems like elliptical galaxies (data derived from Table 3 of Della Valle et al. 1994 and Eq. (1) of Mannucci et al. 2005), we derive a rate of about 1.1 novae $\mathrm{yr}^{-1}$ for the M 31 GC system, which corresponds to about 0.002 novae $\mathrm{yr}^{-1}$ $\mathrm{GC}^{-1}$. This figure is comparable (within a factor of 2) with the observed rate (0.004) of the M 87 GC system obtained by Shara et al. (2004) and suggests that the discovery of only one nova in the M 31 GC system (Shafter \& Quimby 2007) over about a century of observations may be the result of a strong observational bias (see Shafter \& Quimby 2007).

\subsection{Globular cluster nova rate - the X-ray point of view}

While the detection of CNe in GC in the optical is strongly hampered by the light from the GC itself, the detection of supersoft

${ }^{8}$ http://www.bo.astro.it/M31/ 
emission from a hydrogen-burning post-nova atmosphere is not affected by this. However, the presence of other X-ray sources in a GC, such as LMXBs, may create a similar problem for the detection of a SSS in that GC. In the following, we discuss the impact of our results on the rate of novae for the M 31 GCs.

If we assume that both SS1 and SS2 are post-novae and assume the duration of the SSS phase to be of the order of $1 \mathrm{yr}$ then we find a nova rate of 0.015 novae $\mathrm{yr}^{-1} \mathrm{GC}^{-1}$ for the 130 GCs from Galleti et al. (2007) in the field of view of our Chandra HRC-I observations (see also Fig. 2 for the Chandra field). The duration of the SSS phase of novae is a critical parameter for a correct estimate of the nova rate. Although there are some novae known to have much longer SSS phases than $1 \mathrm{yr}$ (see e.g. Ögelman et al. 1993) it is likely (see Pietsch et al. 2007b) that novae with short SSS phases were just not found in the past due to selection effects. Also note, that the SSS phase of SS2 lasted only $\lesssim 4$ months and therefore the assumed duration of $1 \mathrm{yr}$ may still be a quite conservative estimate.

The nova rate computed using the X-ray data on the SSSs is about a factor 10 larger (after assuming the duration of the SSS phase to be $1 \mathrm{yr}$ ) than the nova rate estimated by assuming that GCs produce novae with a rate comparable to the old stellar populations in Ellipticals. The match between the rates in X-ray and in optical is obtained for a duration of the SSS phase of about a decade. Such a long time has been observed indeed (Ögelman et al. 1993), but it does not seem to be the rule (Pietsch et al. 2007b).

The results based on the small size of our sample of GC SSSs are clearly dominated by Poissonian statistics. In this case, the discovery of two GC novae would still be consistent with a nova rate of $\sim 0.002$ novae $\mathrm{yr}^{-1} \mathrm{GC}^{-1}$ (95\% confidence level) and $\sim 0.004$ novae $\mathrm{yr}^{-1} \mathrm{GC}^{-1}$ (99.87\% confidence level), respectively. Both values are below the upper limit of 0.005 novae $\mathrm{yr}^{-1}$ $\mathrm{GC}^{-1}$ estimated by Tomaney et al. (1992) from optical observations.

Furthermore, we want to emphasize the fact that not all optical novae in the Galaxy and in M 31 were detected as SSSs. Novae with supersoft X-ray emission are a subset, with yet unknown size, of the whole nova population. The nova rates deduced from X-ray observations are therefore lower limits on the actual nova rates.

\subsection{Conclusions}

The nova rate in the M 31 GC system may be larger by about one order of magnitude than expected in old stellar systems like giant ellipticals. This fact implies the existence of some other (dynamical) mechanism which acts inside the GCs and is capable to raise the fraction of $\mathrm{CN}$ systems naturally produced by stellar evolution. It is well known that LMXBs are highly overabundant in GCs, with respect to the rest of a galaxy (see e.g. Fan et al. 2005, and references therein). This is explained by dynamical effects like tidal captures of low-mass main-sequence stars by neutron stars (see also Clark 1975; Fabian et al. 1975). One would expect similar effects for WDs (Hut \& Verbunt 1983), but although large numbers of cataclysmic variables (CVs) have been found in Galactic GCs, the number of novae detected in GCs is still small (see Shara et al. 2004, and references therein).

Further, we note that novae with short SSS states seem to be an important contributor to the SSS population of galaxies (see also Pietsch et al. 2007b). XMM-Newton observations of M 31 in the past (Stiele et al. 2008; Pietsch et al. 2005b; Trudolyubov \& Priedhorsky 2004) did not detect SSS with positions that agree with GC positions. This might in part be due to the fact that the individual observations of these monitorings were separated by half a year or more. The discoveries of SS1 and SS2 are the first results of a new monitoring strategy, that uses XMMNewton and Chandra observations that are separated just by 10 days. Future observations that follow this strategy may increase the statistics for SSS in M 31 GCs. If future surveys of M 31 will be able to find between six to eight new GC novae per year, the existence of a nova rate excess, of the size reported above, will be proven at $95 \%$ and $99.87 \%$ confidence level, respectively.

Finally, given the possible link between SSS and type Ia supernovae (SNe-Ia) (e.g. Della Valle \& Livio 1994; Di Stefano \& Rappaport 1994) if a high rate for SSS in GCs will be confirmed by future surveys, it may not be inconceivable to expect detection of SNe-Ia in GC systems around giant ellipticals or bulge dominated galaxies.

Acknowledgements. We wish to thank the referee, Jan-Uwe Ness, for his constructive comments which helped to improve the manuscript significantly. The X-ray work is based in part on observations with XMM-Newton, an ESA Science Mission with instruments and contributions directly funded by ESA Member States and NASA. The XMM-Newton project is supported by the Bundesministerium für Wirtschaft und Technologie / Deutsches Zentrum für Luft- und Raumfahrt (BMWI/DLR FKZ 50 OX 0001), the Max-Planck Society and the Heidenhain-Stiftung. We would like to thank the Swift team for the scheduling of the ToO observations. This research has made use of the NASA/IPAC Extragalactic Database (NED) which is operated by the Jet Propulsion Laboratory, California Institute of Technology, under contract with the National Aeronautics and Space Administration. This study made use of the Digitized Sky Surveys which were produced at the Space Telescope Science Institute under U.S. Government grant NAG W-2166. M. Henze and H.S. acknowledge support from the BMWI/DLR, FKZ 50 OR 0405. G.S. acknowledges support from the BMWI/DLR, FKZ 50 OR 0405, and from grants AYA200804211-C02-01 and AYA2007-66256. M. Hernanz acknowledges support from grants ESP2007-61593 and 2005-SGR00378.

\section{References}

Akerlof, C. W., Kehoe, R. L., McKay, T. A., et al. 2003, PASP, 115, 132

Allen, C. W. 1976, Astrophysical Quantities (Astrophysical Quantities, London: Athlone (3rd edition), 1976)

Anders, E., \& Grevesse, N. 1989, Geochim. Cosmochim. Acta, 53, 197

Balucinska-Church, M., \& McCammon, D. 1992, ApJ, 400, 699

Barmby, P., \& Huchra, J. P. 2000, ApJ, 531, L29

Capaccioli, M., Della Valle, M., Rosino, L., \& D’Onofrio, M. 1989, AJ, 97, 1622

Ciardullo, R., Ford, H. C., Neill, J. D., Jacoby, G. H., \& Shafter, A. W. 1987, ApJ, 318, 520

Ciardullo, R., Shafter, A. W., Ford, H. C., et al. 1990a, ApJ, 356, 472

Ciardullo, R., Tamblyn, P., \& Phillips, A. C. 1990b, PASP, 102, 1113 Clark, G. W. 1975, ApJ, 199, L143

Cohen, J. G. 1988, in The Extragalactic Distance Scale, ed. S. van den Bergh, \& C. J. Pritchet, ASP Conf. Ser., 4, 114

Della Valle, M., \& Livio, M. 1994, ApJ, 423, L31

Della Valle, M., \& Livio, M. 1995, ApJ, 452, 704

Della Valle, M., Rosino, L., Bianchini, A., \& Livio, M. 1994, A\&A, 287, 403

Di Mille, F., Ciroi, S., Orio, M., et al. 2008, The Astronomer's Telegram, 1703, 1

Di Stefano, R., \& Rappaport, S. 1994, ApJ, 423, 274

Dotani, T., Asai, K., \& Greiner, J. 1999, PASJ, 51, 519

Edmonds, P. D., Kahabka, P., \& Heinke, C. O. 2004, ApJ, 611, 413

Elvis, M., Civano, F., Vignali, C., et al. 2009, ArXiv e-prints

Fabian, A. C., Pringle, J. E., \& Rees, M. J. 1975, MNRAS, 172, 15P

Fan, Z., Ma, J., Zhou, X., et al. 2005, PASP, 117, 1236

Freedman, W. L., Madore, B. F., Gibson, B. K., et al. 2001, ApJ, 553, 47

Galache, J. L., Garcia, M. R., Torres, M. A. P., et al. 2007, The Astronomer's Telegram, 1328, 1

Galleti, S., Federici, L., Bellazzini, M., Fusi Pecci, F., \& Macrina, S. 2004, A\&A, 416, 917

Galleti, S., Bellazzini, M., Federici, L., \& Fusi Pecci, F. 2005, A\&A, 436, 535

Galleti, S., Federici, L., Bellazzini, M., Buzzoni, A., \& Fusi Pecci, F. 2006, A\&A, 456, 985

Galleti, S., Bellazzini, M., Federici, L., Buzzoni, A., \& Fusi Pecci, F. 2007, A\&A, 471, 127

Greiner, J., Hasinger, G., \& Kahabka, P. 1991, A\&A, 246, L17 
Haberl, F., Henze, M., \& Pietsch, W. 2007a, The Astronomer's Telegram, 1296, 1 Haberl, F., Pietsch, W., \& Henze, M. 2007b, The Astronomer's Telegram, 1306, 1 Hachisu, I., \& Kato, M. 2006, ApJS, 167, 59

Henze, M., Burwitz, V., Pietsch, W., et al. 2008a, The Astronomer's Telegram, 1654,1

Henze, M., Meusinger, H., \& Pietsch, W. 2008b, A\&A, 477, 67

Hernanz, M. 2005, in The Astrophysics of Cataclysmic Variables and Related Objects, ed. J.-M. Hameury, \& J.-P. Lasota, ASP Conf. Ser., 330, 265

Hertz, P., \& Grindlay, J. E. 1983, ApJ, 267, L83

Holland, S. 1998, AJ, 115, 1916

Hubble, E. P. 1929, ApJ, 69, 103

Hut, P., \& Verbunt, F. 1983, Nature, 301, 587

Jose, J., \& Hernanz, M. 1998, ApJ, 494, 680

Kahabka, P., \& van den Heuvel, E. P. J. 1997, ARA\&A, 35, 69

Kong, A. K. H., \& Di Stefano, R. 2007a, The Astronomer's Telegram, 1334, 1

Kong, A. K. H., \& Di Stefano, R. 2007b, The Astronomer's Telegram, 1333, 1

Krautter, J. 2002, in Classical Nova Explosions, ed. M. Hernanz, \& J. José, ASP Conf. Ser., 637, 345

Landsman, W. B. 1989, BAAS, 21, 784

Maccacaro, T., Gioia, I. M., Wolter, A., Zamorani, G., \& Stocke, J. T. 1988, ApJ, 326,680

Mannucci, F., Della Valle, M., Panagia, N., et al. 2005, A\&A, 433, 807

Massey, P., Olsen, K. A. G., Hodge, P. W., et al. 2006, AJ, 131, 2478

Monet, D. G., Levine, S. E., Canzian, B., et al. 2003, AJ, 125, 984

Ness, J.-U., Starrfield, S., Beardmore, A. P., et al. 2007, ApJ, 665, 1334

Ögelman, H., Orio, M., Krautter, J., \& Starrfield, S. 1993, Nature, 361, 331

Parmar, A. N., Kahabka, P., Hartmann, H. W., et al. 1997, A\&A, 323, L33

Parmar, A. N., Kahabka, P., Hartmann, H. W., Heise, J., \& Taylor, B. G. 1998, A\&A, 332, 199

Payne-Gaposchkin, C. 1964, The galactic novae (New York: Dover Publication, 1964)

Perlmutter, S., Aldering, G., Goldhaber, G., et al. 1999, ApJ, 517, 565

Pietsch, W., Fliri, J., Freyberg, M. J., et al. 2005a, A\&A, 442, 879

Pietsch, W., Freyberg, M., \& Haberl, F. 2005b, A\&A, 434, 483

Pietsch, W., Burwitz, V., Greiner, J., et al. 2007a, The Astronomer's Telegram, 1294, 1
Pietsch, W., Haberl, F., Sala, G., et al. 2007b, A\&A, 465, 375

Pietsch, W., Freyberg, M., Haberl, F., Henze, M., \& Stiele, H. 2008a, The Astronomer's Telegram, 1647, 1

Pietsch, W., Freyberg, M., Henze, M., Stiele, H., \& Immler, S. 2008b, The Astronomer's Telegram, 1671, 1

Quimby, R. 2006, Ph.D. Thesis, University of Texas, United States

Rauch, T. 1997, A\&A, 320, 237

Rauch, T. 2003, A\&A, 403, 709

Rauch, T., \& Deetjen, J. L. 2003, in Stellar Atmosphere Modeling, ed. I. Hubeny, D. Mihalas, \& K. Werner, ASP Conf. Ser., 288, 103

Sala, G., \& Hernanz, M. 2005, A\&A, 439, 1061

Schlegel, D. J., Finkbeiner, D. P., \& Davis, M. 1998, ApJ, 500, 525

Shafter, A. W. 1997, ApJ, 487, 226

Shafter, A. W., \& Quimby, R. M. 2007, ApJ, 671, L121

Shara, M. M., Zurek, D. R., Baltz, E. A., Lauer, T. R., \& Silk, J. 2004, ApJ, 605, L117

Stanek, K. Z., \& Garnavich, P. M. 1998, ApJ, 503, L131

Stark, A. A., Gammie, C. F., Wilson, R. W., et al. 1992, ApJS, 79, 77

Starrfield, S. 1989, in Classical Novae, 39

Starrfield, S., Sparks, W. M., \& Truran, J. W. 1974, ApJS, 28, 247

Stetson, P. B. 1987, PASP, 99, 191

Stiele, H., Pietsch, W., Haberl, F., \& Freyberg, M. 2008, A\&A, 480, 599

Tomaney, A., Crotts, A., \& Shafter, A. 1992, in BAAS, 24, 1237

Trudolyubov, S., \& Priedhorsky, W. 2004, ApJ, 616, 821

Tuchman, Y., \& Truran, J. W. 1998, ApJ, 503, 381

van den Bergh, S., \& Younger, P. F. 1987, A\&AS, 70, 125

Verbunt, F., Bunk, W., Hasinger, G., \& Johnston, H. M. 1995, A\&A, 300, 732

Warner, B. 1995, Cataclysmic variable stars (Cambridge Astrophysics Series, Cambridge, New York: Cambridge University Press, 1995)

Williams, G. G., Milne, P. A., Park, H. S., et al. 2008, in Am. Inst. Phys. Conf. Ser., 1000, 535

Williams, R. E. 1992, AJ, 104, 725

Wilms, J., Allen, A., \& McCray, R. 2000, ApJ, 542, 914

Yaron, O., Prialnik, D., Shara, M. M., \& Kovetz, A. 2005, ApJ, 623, 398 